\title{
Platelet ascorbic acid levels in normal subjects and in disease
}

\author{
J. V. LLOYD, P. S. DAVIS, H. EMERY, AND H. LANDER \\ From the Department of Medicine, University of Adelaide, Adelaide, South Australia
}

SYNOPSIS The platelet ascorbic acid concentration was measured in 26 normal subjects and found to be 20 times as high as in plasma. This is in agreement with previous reports in the literature. The platelets of patients with uraemia, leukaemia, and megaloblastic anaemia had a lower than normal platelet ascorbic acid content. In uraemia and megaloblastic anaemia the plasma ascorbic acid concentration was normal suggesting that a platelet defect may be responsible for the low platelet ascorbic acid content. In leukaemia the low platelet ascorbic acid content is probably secondary to a low plasma level.

A number of observations support the contention that ascorbic acid is of importance in the metabolism of blood platelets. It is known that the ascorbic acid content of platelets is high (Barkhan and Howard, 1958) and that platelet adhesiveness to glass is reduced in scurvy both in man (Wilson, McNichol, and Douglas, 1967) and in guinea pigs (Born and Wright, 1967). Furthermore, Sahud and Aggeler (1970) have shown that there is a relationship between platelet aggregation induced by thrombin, epinephrine, collagen, or ADP and the utilization of ascorbic acid by platelets. Hence, although the haemorrhagic diathesis of scurvy has been held to be due to a capillary defect (Bartley, Krebs, and O'Brien, 1953), it is probable that a platelet defect contributes significantly to the deficiency in haemostasis.

In a search for a possible role for platelet ascorbic acid in disease, we have measured the platelet ascorbic acid content in healthy subjects and in patients with various disorders. We report here on the values found in uraemia, leukaemia, and megaloblastic anaemia.

\section{Methods}

Platelet ascorbic acid content was estimated as described previously (Lloyd, Davis, and Lander, 1969). Plasma ascorbic acid concentration was measured by the dinitrophenylhydrazine procedure (Roe, 1954). All estimations were performed on blood freshly collected two to two and a half hours after a light breakfast. All subjects were questioned to establish the presence or absence of a bleeding Received for publication 27 October 1971. disorder in themselves or their families. They were $\vec{\varphi}$ also questioned concerning their smoking habits and $N$ diet with particular respect to foods containing ascorbic acid.

\section{Results}

NORMAL SUBJECTS

Twenty-six subjects were studied. Of these, 12 were males aged 19-28 and 14 were females aged 18-20. The mean platelet ascorbic acid content was $3 \cdot 5 \pm$ $1.4 \mu \mathrm{g} / 10^{\circ}$ cells. This can be compared with $2.91 \mu \mathrm{g} / 10^{9}$ cells (Barkhan and Howard, 1958) and $6.28 \mu \mathrm{g} / 10^{\circ}$ cells (Sahud and Aggeler, 1970); but these authors used the dinitrophenylhydrazine procedure which has been shown to overestimate the ascorbic acid content of tissues (Lloyd et al, 1969; Zloch and Ginter, 1970).

The mean plasma ascorbic acid content of our normal subjects was $0.79 \pm 0.32 \mu \mathrm{g} / 100 \mathrm{ml}$, which is consistent with previous reports (Goldsmith, 1961). The mean platelet ascorbic acid content in or these subjects was $0.16 \mu \mathrm{g} / \mathrm{mg}$. Platelet ascorbic acid $N$ concentration was therefore approximately 20 times that of plasma.

URAEMIA

Thirteen patients suffering from uraemia were studied. The blood urea nitrogen ranged from 32 to $132 \mathrm{mg} / 100 \mathrm{ml}$. Four patients were being treated by haemodialysis twice weekly, and in these subjects blood was taken for estimation just before dialysis.

The platelet ascorbic acid content was $2.0 \pm$ $2.0 \mu \mathrm{g} / 10^{\circ}$ cells which is significantly $(P=0.01)$ 478 

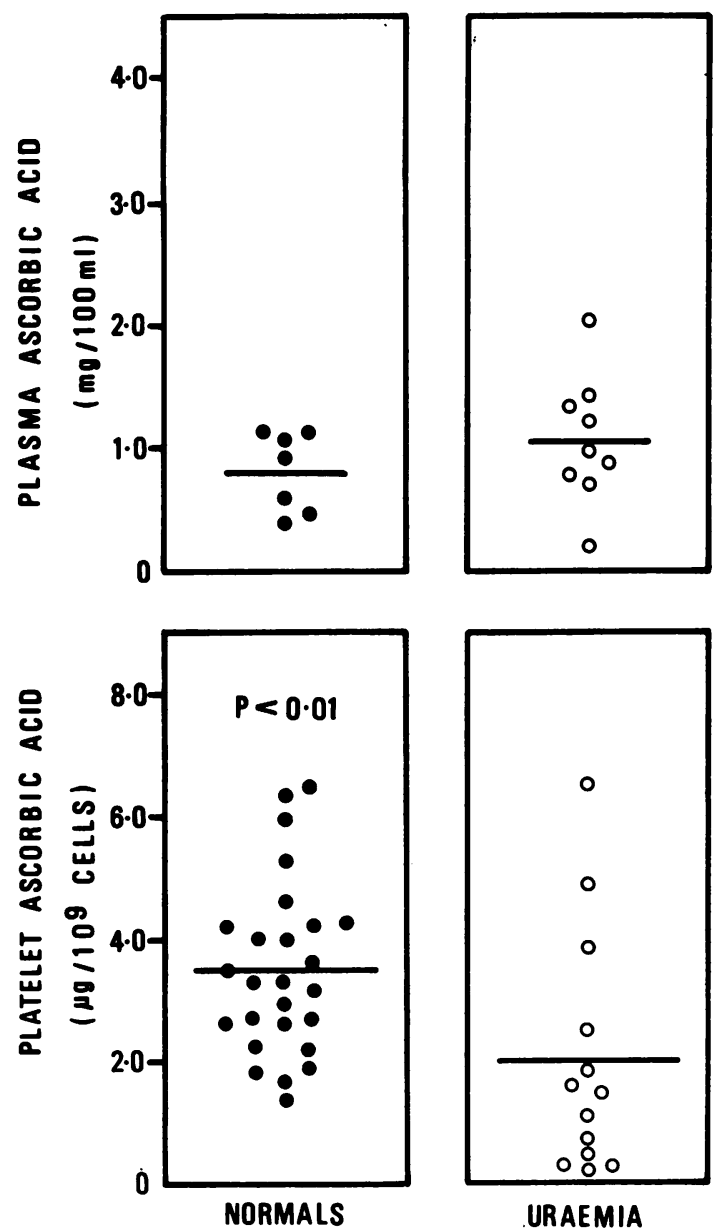

Fig. 1.

Fig. 1 Platelet and plasma ascorbic acid concentrations in patients with uraemia compared with the normal ranges. Mean values are marked by a horizontal dash.

Fig. 2 Effect of large doses of ascorbic acid on the platelet and plasma ascorbic acid concentrations in patients with uraemia. The hatched areas represent the normal ranges (mean \pm 1 standard deviation).

lower than normal (Fig. 1). Since the distribution of platelet ascorbic acid content in uraemia was somewhat skew the statistical significance of the result was confirmed by the rank-sum test $(P<0.01)$ (Dixon and Massey, 1969). The mean plasma concentration, however, did not differ significantly from normal, the value actually being somewhat higher than in normal subjects.

Five of the uraemic patients were studied before
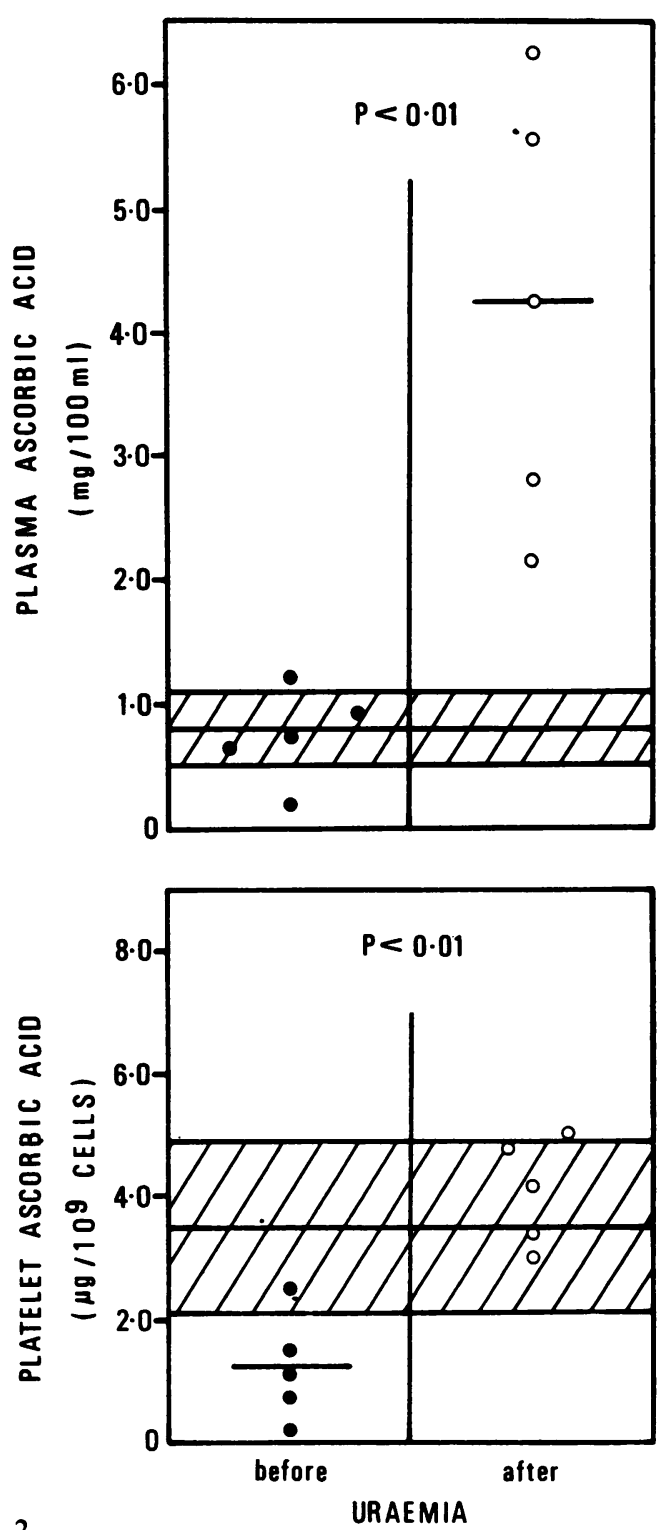

Fig. 2.

and after the administration of large doses of ascorbic acid ( $1.0 \mathrm{~g}$ daily for four days). This dose is sufficient to saturate the tissues of normal subjects (Cuttle, 1938). Before the administration of ascorbic acid the platelet ascorbic acid content was below the normal range $(0.005>P>0.001)$ but the plasma concentration was well within normal limits (Fig. 2). After the administration of ascorbic acid the platelet ascorbic acid content became normal, whereas 

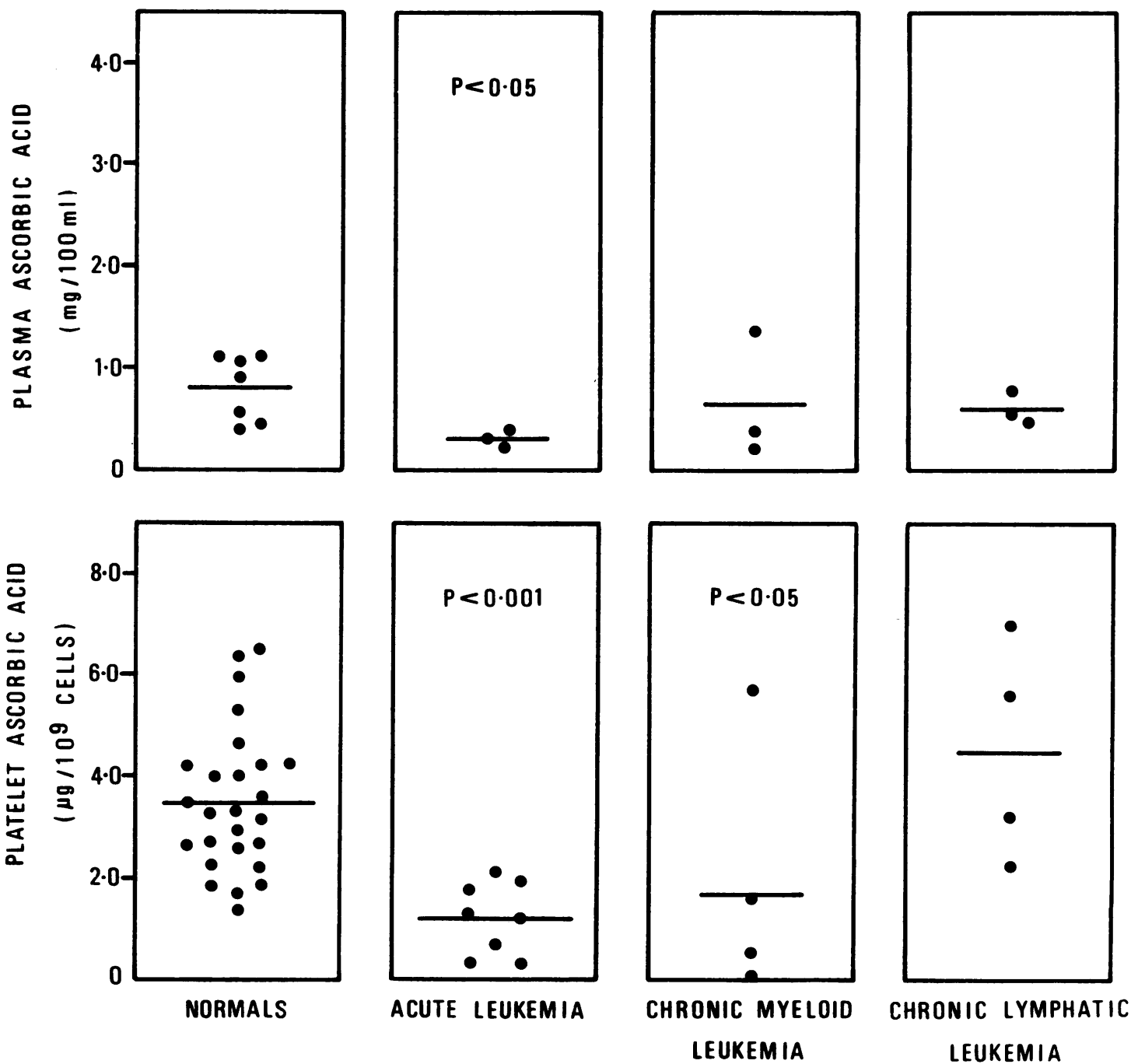

Fig. 3 Platelet and plasma ascorbic acid concentrations in patients with leukaemia compared with the normal ranges. Mean values are marked by a horizontal dash.

plasma levels rose to values much higher than those of $1.0-1.4 \mathrm{mg} / 100 \mathrm{ml}$ obtained on the administration of these doses to normal subjects (Goldsmith, 1961).

\section{LEUKAEMIA}

Sixteen patients were studied. Of these eight were suffering from acute leukaemia, four from chronic myeloid leukaemia, and four from chronic lymphatic leukaemia. Of the cases of acute leukaemia, four were aleukaemic and one was in haematological remission. While all four cases of chronic myeloid leukaemia were in an active phase of the disease, one of the patients with chronic lymphatic leukaemia was in haematological remission.

The mean platelet ascorbic acid content in the patients with acute leukaemia was $1.2 \pm 0.7 \mu \mathrm{g} / 10^{\circ}$ cells, which is significantly lower than normal (Fig. 3). The plasma ascorbic acid concentration, measured in three of the subjects, was also significantly reduced.

In patients with chronic myeloid leukaemia, despite the high value obtained in one subject who was on a high intake of fruit juice, the platelet ascorbic acid content was significantly lower than 
Platelet ascorbic acid levels in normal subjects and in disease
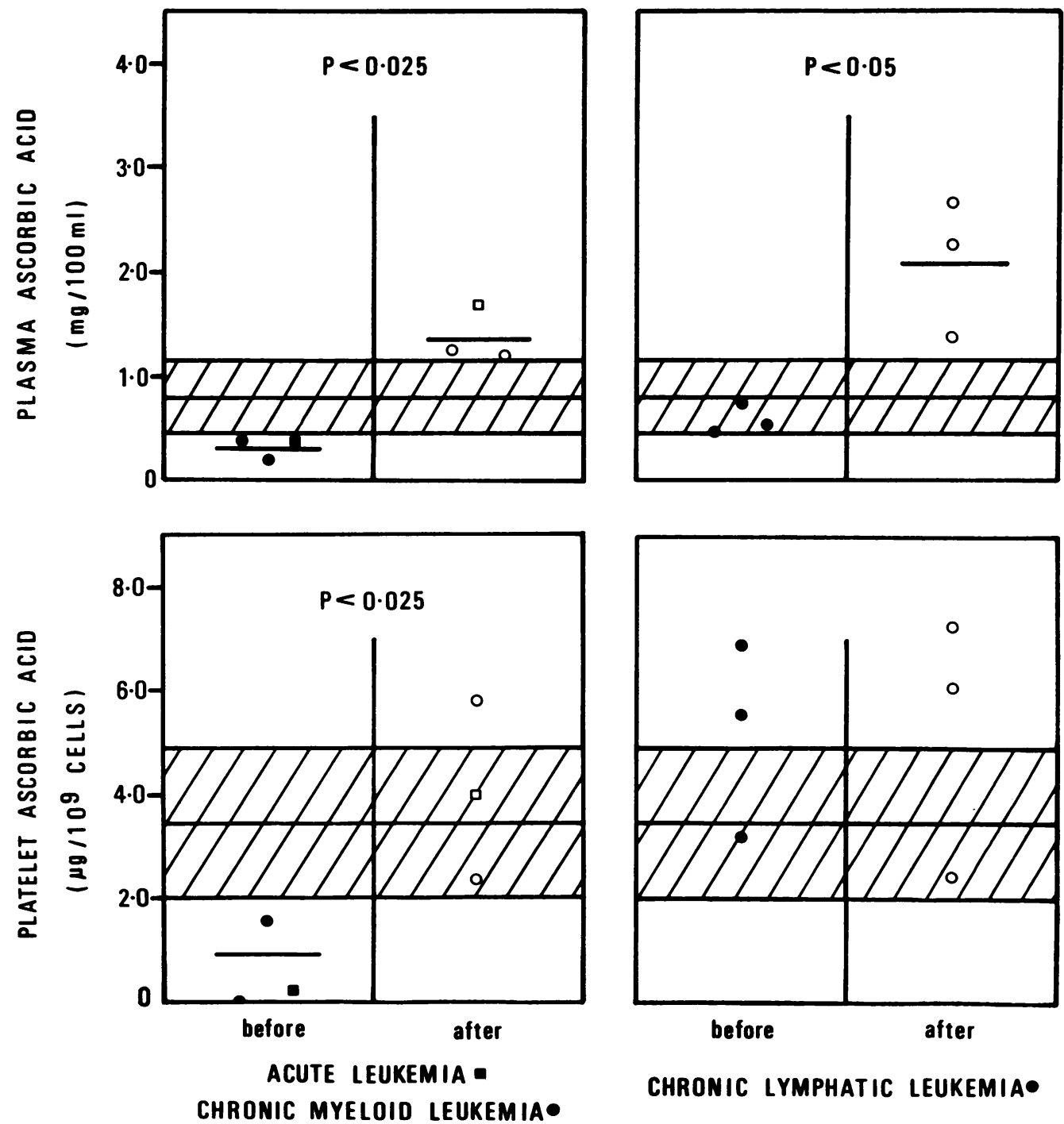

Fig. 4 Effect of large doses of ascorbic acid on the platelet and plasma ascorbic acid concentrations in patients with leukaemia. The hatched areas represent the normal ranges (mean \pm 1 standard deviation).

normal (Fig. 3). In patients with chronic lymphatic leukaemia, however, the platelet ascorbic acid content was not significantly different from normal (Fig. 3).

Six of the cases of leukaemia were studied again after administration of $1.0 \mathrm{~g}$ of ascorbic acid daily for four days. The plasma ascorbic acid concentrations rose to values which are consistent with values found in normal subjects after the administration (Goldsmith, 1961) of such large doses of ascorbic acid (Fig. 4).
In the three patients with leukaemia with low ascorbic acid concentrations before the experiment, the platelet ascorbic acid content returned to the normal range. In the three with chronic lymphatic leukaemia there were no changes seen in platelet ascorbic acid content (Fig. 4).

\section{MEGALOBLASTIC ANAEMIA}

Five patients, all of whom had megaloblastic changes on bone marrow biopsy, were studied. Three of these patients suffered from pernicious anaemia, and 

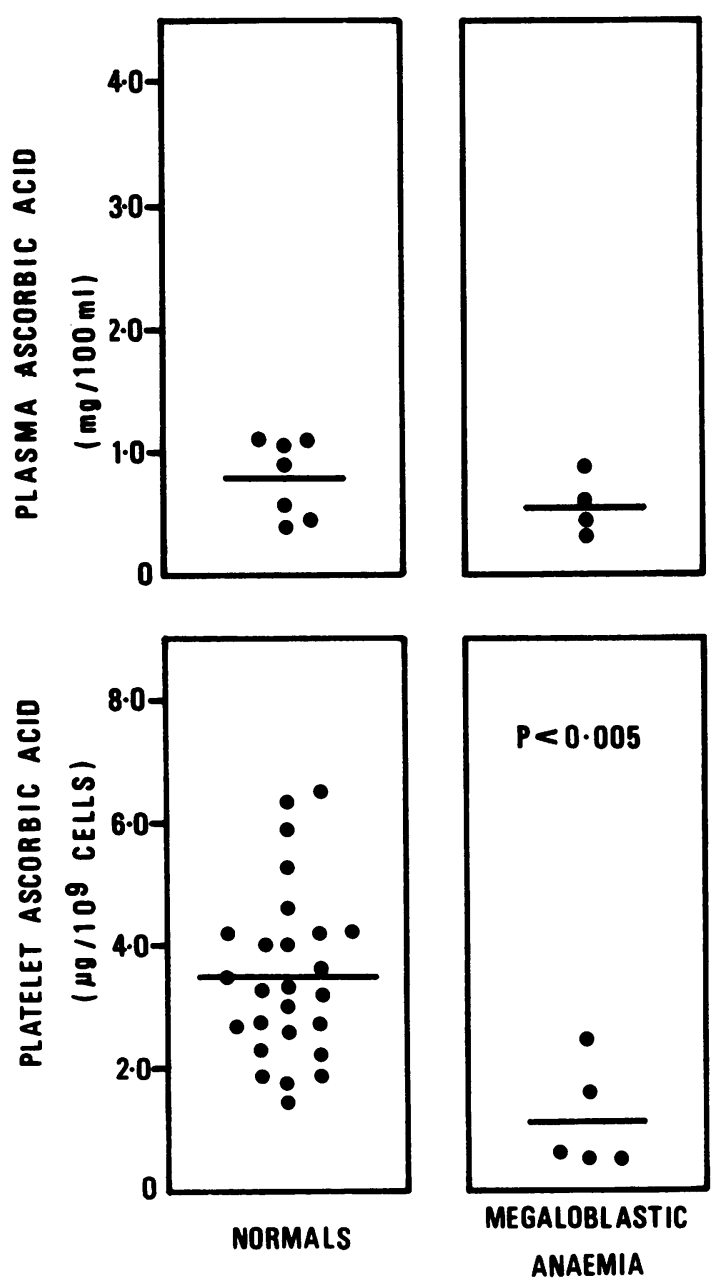

Fig. 5 Platelet and plasma ascorbic acid concentrations in patients with megaloblastic anaemia compared with the normal ranges. Mean values are marked by a horizontal dash.

one from folic acid deficiency. The fifth patient had had a partial gastrectomy, was being treated with phenytoin for epilepsy, and had low normal serum concentrations of both vitamin $\mathbf{B}_{12}$ and folic acid.

The mean platelet ascorbic acid content was markedly reduced despite a normal mean plasma concentration (Fig. 5). In one patient receiving vitamin $\mathbf{B}_{12}$ therapy for pernicious anaemia the platelet ascorbic acid concentration increased from $0.5 \mu \mathrm{g} / 10^{\circ}$ platelets to $5.2 \mu \mathrm{g} / 10^{\circ}$ platelets over a period of two weeks with no other ascorbic acid intake than that supplied in a ward diet.
J. V. Lloyd, P. S. Davis, H. Emery, and H. Lander

\section{Discussion}

The high ascorbic acid content of platelets suggests that there is a mechanism for maintaining a gradient of concentration between the plasma and the platelets. Hence, a low platelet ascorbic acid concentration might result either from a low plasma ascorbic acid level (which may be dietary in origin) or from a defect in the ability of the platelet to concentrate ascorbic acid. In uraemia, this concentrating mechanism appears to be defective, for the platelet ascorbic acid content is low in the presence of a normal plasma ascorbic acid concentration. In megaloblastic anaemia this also appears to be the case, for the platelet content of ascorbic acid is on lowered to a much greater extent than the slight (and statistically insignificant) fall in the plasma ascorbic acid concentration. It is unlikely that an ascorbic acid-deficient diet is responsible for the low platelet ascorbic acid content in uraemia and in megaloblastic anaemia, for it is known that such a diet leads first to depletion of plasma ascorbic acid and only after some time to depletion of tissue stores as $\vec{\oplus}$ estimated by the buffy layer ascorbic acid value $N$ (Crandon, Lund, and Dill, 1940; Bartley et al, 1953).

In acute leukaemia, on the other hand, the ascorbic acid concentration in platelets is probably secondary to the low plasma ascorbic acid concentration and not to a defect in the platelets. It is possible that the low plasma levels found are dietary in origin for, although no marked dietary ascorbic acid deficiency was noted in these patients on dietary history, it is difficult to exclude such a deficiency without an ascorbic acid analysis of all food consumed.

The values for platelet ascorbic acid content in uraemia and in leukaemia were not so low as to be $\delta$ consistent with the zero levels found in the buffy layer of centrifuged blood from patients with scurvy. 을 Crandon et al (1940) and Bartley et al (1953) found in human depletion experiments that the buffy layer and plasma ascorbic acid concentrations reached zero for their method some time before the or onset of bleeding manifestations. It is unlikely, $N$ therefore, that low platelet ascorbic acid levels contribute significantly to the bleeding tendency found in patients suffering from uraemia or acute leukaemia.

The disproportionate lowering of the ascorbic acid $\stackrel{\mathbb{P}}{\rightarrow}$ content of platelets compared to plasma in uraemia $T$ and in megaloblastic anaemia may reflect a defect in $\overrightarrow{0}$ tissue stores of ascorbic acid in these disorders. The $\stackrel{\vec{P}}{\vec{P}}$ buffy layer ascorbic acid content is held to be a $\stackrel{\mathbb{D}}{\mathbb{Q}}$ measure of general tissue stores (Crandon et al, $\stackrel{\circ}{\sigma}$ 1940; Bartley et al, 1953), and since the buffy 
layer consists of both white cells and platelets which contain about equal concentrations of ascorbic acid (Barkhan and Howard, 1958) it is likely that platelet ascorbic acid content also parallels tissue stores.

The defect in the platelet mechanism for concentrating ascorbic acid in uraemia and megaloblastic anaemia is interesting in view of other defects in platelet function which have been described in these disorders. Platelets from patients with uraemia have been shown to aggregate less well in response to ADP (Castaldi, Rozenberg, and Stewart, 1966) and to be less active in the thromboplastin generation test (Cheney and Bonnin, 1962) than normal platelets. Platelets from patients with pernicious anaemia have been reported to have a defective thromboplastic function (Stefanini and Karaca, 1966), although the decreased phospholipid content reported in the same paper suggests that this may be due to the platelets being smaller than normal.

There are no previous reports on platelet ascorbic acid content in uraemia and in megaloblastic anaemia. In leukaemia, however, platelets have been subjected to ascorbic acid analysis by Barkhan and Howard (1958). They also found a low plasma ascorbic acid concentration in leukaemia but no decrease in platelet ascorbic acid concentration. Waldo and Zipf (1955) found, however, that the ascorbic acid content of both the plasma and the buffy layer was low in patients with leukaemia. The reason for the conflicting results may be related either to dietary factors or to differences in the severity of the disease in the subjects studied.

A relationship between ascorbic acid status and megaloblastic anaemia has already been demonstrated. May, Hamilton, and Stewart (1952) have shown in monkeys that folic acid deficiency more readily induces megaloblastic anaemia if the animals are also deficient in ascorbic acid. Ascorbic acid deficiency has been shown to be the cause of folic acid-resistant megaloblastic anaemia (Cox, Gaddie, Matthews, Cooke, and Meynell, 1967). Patients with vitamin $\mathbf{B}_{12}$ deficiency have been reported to have a low serum ascorbic acid level which is difficult to correct unless vitamin $\mathbf{B}_{12}$ stores are first replenished (Cox et al, 1958; Kahn and Brodsky, 1966). The plasma ascorbic acid levels in megaloblastic anaemia found in the present study might have proven to be significantly reduced if more patients had been available for study.

This work was performed under a grant in aid from the National Heart Foundation of Australia.

\section{References}

Barkhan, P., and Howard, A. N. (1958). Distribution of ascorbic acid in normal and leukaemic human blood. Biochem. J., 70, 163-168.

Bartley, W., Krebs, H. A., and O'Brien, J. R. P. (1953). Vitamin C requirement of human adults: a report by the Vitamin $C$ Subcommittee of the Accessory Food Factors Committee (M.R.C.), Spec. Rep. Ser. med. Res. Coun. (Lond.), 280.

Born, G. V. R., and Wright, H. P. (1967). Platelet adhesiveness in experimental scurvy. Lancet, 1, 477-478.

Castaldi, P. A., Rozenberg, M. C., and Stewart, J. H. (1966). The bleeding disorder of uraemia. Lancet, 2, 66-69.

Cheney, K., and Bonnin, J. A. (1962). Haemorrhage, platelet dysfunction and other coagulation defects in uraemia. Brit. $J$. Haemat., 8, 215-222.

Cox, E. V., Gaddie, R., Matthews, D., Cooke, W. T., and Meynell, M. J. (1958). An inter-relationship between ascorbic acid and cyanocobalamin. Clin. Sci., 17, 681-692.

Crandon, J. H., Lund, C. C., and Dill, D. B. (1940). Experimental human scurvy. New Engl. J. Med., 223, 353-369.

Cuttle, T. D. (1938). Observations on relation of leucocytosis to ascorbic acid requirements. Quart. J. Med., 7, 575-584.

Dixon, W. J., and Massey, F. J. (1969). Introduction to Statistical Analysis, 3rd ed.,p. 344. McGraw-Hill, New York.

Goldsmith, G. A. (1961). Human requirements for vitamin $\mathbf{C}$ and its use in clinical medicine. Ann. N.Y. Acad. Sci., 92, 230-245.

Kahn, S. B., and Brodsky, I. (1966). Vitamin $B_{12}$, ascorbic acid and iron metabolism in scurvy. Amer. J. Med., 40, 119-126.

Lloyd, J. V., Davis, P. S., and Lander, H. (1969). New method for the estimation of platelet ascorbic acid. J. clin. Path., 22, 453-457.

May, C. D., Hamilton, A., and Stewart, C. T. (1952). Experimental megaloblastic anemia and scurvy in the monkey. IV. Vitamin $B_{12}$ and folic acid compounds on the diet, liver, urine and feces and effects of therapy. Blood, 7, 978-991.

Roe, J. H. (1954). Chemical determination of ascorbic, dehydroascorbic and diketogulonic acids. Meth. Biochem., Anal., 1, 115-139.

Sahud, M. A., and Aggeler, P. M. (1970). Utilization of ascorbic acid during platelet aggregation. Proc. Soc. exp. Biol. (N.Y.), 134, 13-16.

Stefanini, M., and Karaca, M. (1966). Acquired thrombocytopathy in patients with pernicious anaemia. Lancet, 1, 400-402.

Waldo, A. L., and Zipf, R. E. (1955). Ascorbic acid level in leukemic patients. Cancer (Philad.), 8, 187-190.

Wilson, P. A., McNicol, G. P., and Douglas, A. S. (1967). Platelet. abnormality in human scurvy. Lancet, 1, 975-978.

Zloch, Z., and Ginter, E. (1970). Thin-layer chromatographic determination of L-ascorbic, L-dehydroascorbic and 2.3-diketoL-gulonic acids in animal tissues, blood and urine. $Z$. klin. Chem., 8, 302-305. 